\title{
Monte Carlo code for the study of the dynamic light field at the wavy atmosphere-ocean interface
}

M. Hieronymi
martin.hieronymi@hzg.de

A radiative transfer model has been developed to study the solar radiation budget at the wave-deflected air-sea interface. The model is used to characterize fluctuations of the underwater light field, i.e. down- and upwelling irradiance, irradiance reflectance, and upwelling radiance just below the surface, subject to changing sun zenith angles and percentages of diffuse sky radiation to the total insolation. The focusing of sunlight is most effective under clear skies; the variability of downwelling irradiance is significantly smaller under overcast conditions. In general, maximum and deep-reaching fluctuations arise at high sun positions, but the behaviour is much more differentiated and exceptions are discussed. Furthermore, wave shadowing effects have been studied; these become increasingly important for low sun elevations. There are indications that the light transmission into water up to now is overestimated for solar zenith angles near the horizon. [DOI: http://dx.doi.org/10.2971/jeos.2013.13039]

Keywords: Radiative transfer model, Monte Carlo method, air-sea interactions, wave shadowing, light field fluctuations

\section{INTRODUCTION}

Sunlight is essential for many biological, physical, and chemical processes in the ocean. The availability of solar radiation in sea water depends on the sun position, the composition of the atmosphere, the structure of the water surface, the inherent optical properties and the depth of the water, and in optically shallow waters also the appearance of the sea bottom. Apart from the obvious reasons as the diurnal cycle of the sun or passing clouds, waves at the sea surface are a major source for variations in the light field, both beneath and above the surface.

Light is refracted at the curved water surface; light beams are focused beneath wave crests and spread below wave troughs accordingly. In the upper meters near the water surface, very intense fluctuations of downwelling irradiance can be observed due to this lensing effect. For example, recent field studies have shown that short-duration irradiance flashes can exceed the tenfold of the mean irradiance in about $1 \mathrm{~m}$ depth [1]. After a short increase, irradiance fluctuations decrease with depth due to light scattering in water and the geometrical superposition of individual focal systems of small-scale waves. The magnitude, the decay, and the spatiotemporal scales of irradiance fluctuations depend on the roughness of the water surface, i.e. wind speed and wave characteristics, and the above mentioned environmental conditions [2]-[13].

But waves not only cause strong fluctuations of the downwelling radiation, they also have impact on the mean value. If light beams are laterally deflected away from the perpendicular plane, less of their radiative contribution can add up to the total downward plane irradiance per depth. Consequently, in a case with more frequent steep wave slopes at the sea surface, i.e. high wind speed, the total downwelling irradiance decreases faster along the water column compared to a case with a flat surface. This effect is further amplified by the fact that in this case more light is reflected at the surface and thus, less radiation actually transmits the air-sea interface. And this implies that the wind-related surface roughness has an effect on the upwelling and water-leaving radiation too.

The wind influence on the roughness of the sea surface was deduced from sun glint pictures by Cox and Munk [14] (sun glint is part of the upwelling radiation above water). Their wind parametrization is applied in many methodologically different radiative transfer models of the aquatic system, e.g. Hydrolight [15] (using the invariant imbedding method), MOMO [16] (matrix-operator method), DISORT [17] or SCIATRAN [18] (both discrete-ordinates method); a comparison of different numerical models is published by Mobley et al. [19]. Using the Cox-Munk wave slope statistics for the realization of random sea surfaces for modelling the optical effects of the wind-blown surface is absolutely sufficient in most situations [20, 21]. However, the approach lacks information on the order of slopes in a wave train and on the variance of the sea surface elevation which is mainly due to larger gravity waves, i.e. the sea state. From this reason, neither wave focusing nor shadowing effects of wave crests can be regarded. Therefore, Mobley [20] restricts the validity of Hydrolight, and thus the usability of wave slope statistics, on solar zenith angles and viewing directions that are not near the horizon. But we should keep in mind that, seen over the course of a year, in fact many sea areas, as for example the North Sea, most frequently have flat irradiation angles. At low sun altitudes, the wave geometry generates clear shadows, which can be seen by eye, the side of the wave crest 
that faces the sun is illuminated and bright, whereas the back side is significantly darker.

In this paper a specialized radiative transfer model is presented that aims at the limitations of previous models regarding light field variability, wave shadowing, and low sun positions. Theoretically, whitecaps at high wind speeds, air bubble injection, and locally assigned scattering and absorption properties can be treated by the model, but this is not shown in the present article. It is a Monte Carlo code with high spatial resolution in two dimensions and consideration of exactly described almost realistic wave profiles that account for capillary to large gravity waves. In fact, the model has some features in common with the Monte Carlo codes by D'Alimonte et al. [22] and $\mathrm{Xu}$ et al. [13]. And there are further comparable recent model studies on underwater light variability and water surface effects (e.g. [23]-[25]). However, the model in this paper is used to show the variability of the underwater light field due to changing solar zenith angles and diffuse sky radiation. An inter-comparison with the radiative transfer model Hydrolight is provided.

\section{RADIATIVE TRANSFER MODEL}

The presented model is a further development of the Monte Carlo (MC) radiative transfer code by Hieronymi et al. [26]. The model was optimized for investigations of wind and surface wave effects on fluctuations of downwelling irradiance in natural waters $[11,27]$. And it was also possible to study the variability of underwater downwelling irradiance in response to changing daylight diffuseness [12]. The purpose of the new model is to simulate the entire underwater light field, including the upwelling irradiance distribution, and to gain insights into the radiative transfer processes at the wavedeflected boundary layer between air and sea. By means of the new approach it now is possible to model the radiation input at solar zenith angles near the horizon and to consider wave shadowing effects.

The underlying model input parameters and boundary conditions are summarized in Table 1 . The simulations are carried out for monochromatic light at a wavelength of $490 \mathrm{~nm}$; a spectral range of high transmittance in sea water and thus with a potentially high depth-impact of wave-caused light variability. The water body has homogeneous inherent optical properties that correspond to a chlorophyll-a concentration $\mathrm{Chl}$ of $0.2 \mathrm{mg} \mathrm{m}^{-3}$, which is roughly the estimated annual mean value of $\mathrm{Chl}$ for the deep global ocean [28]. The corresponding total attenuation coefficient $c$, which is $a+b$, is $0.1551 \mathrm{~m}^{-1}$. The used global scattering phase function for oceanic waters that account for both particles and water-molecular is based on Petzold's work [29]. The model ignores a diversity of factors that may influence the light field. These include inelastic scattering processes, polarization effects, optical stratification of the water column, whitecaps of breaking waves, and air bubbles. The two-dimensional simulation domain has a horizontally grid resolution of $1 \mathrm{~cm}$; the wave resolution is two orders of magnitude smaller than that used for the model domain. With this respect, we have to keep in mind that there

\begin{tabular}{|c|c|c|c|}
\hline Wavelength of light & $\lambda$ & {$[\mathrm{nm}]$} & 490 \\
\hline Refractive index of seawater & $N$ & {$[\mathrm{-}]$} & 1.34 \\
\hline Total absorption coefficient & $a$ & {$[\mathrm{~m}-1]$} & 0.0349 \\
\hline Total scattering coefficient & $b$ & {$[\mathrm{~m}-1]$} & 0.1202 \\
\hline $\begin{array}{c}\text { Maximum number of } \\
\text { scattering events }\end{array}$ & $N_{\max }$ & {$[-$ ] } & 47 \\
\hline Horizontally wave resolution & $d x_{w}$ & {$[\mathrm{~m}]$} & 0.0001 \\
\hline Horizontally grid resolution & $d x$ & {$[\mathrm{~m}]$} & 0.01 \\
\hline Vertically grid resolution & $d z$ & {$[\mathrm{~m}]$} & 0.05 \\
\hline Grid width & $x$ & {$[\mathrm{~m}]$} & 10 \\
\hline Maximum water (grid) depth & $z_{\max }$ & {$[\mathrm{m}]$} & 40 \\
\hline
\end{tabular}

TABLE 1 Underlying data for radiative transfer simulations.

are effects of the model resolution, or the size of the irradiance sensor respectively, on the light field statistics [1, 26].

\subsection{Air-sea interface}

The sea surface can be freely selected. In this study, a slight sea state with a significant wave height $H_{s}$ of $1 \mathrm{~m}$ and a peak period $T_{p}$ of $4 \mathrm{~s}$ with a moderate breeze of $6 \mathrm{~m} \mathrm{~s}^{-1}$ has been selected. Such sea conditions frequently occur. The wave profile is generated from a sea spectrum for long and short waves (details are given in [11]). Vertical deflections of the two-dimensional wave profile are taken into full consideration. The slope distribution approximately fits to the corresponding Cox-Munk distribution, which is used in various radiative transfer models, e.g. Hydrolight [15]. Figure 1 shows a $100 \mathrm{~m}$ long wave profile with its distribution of slope angles and their associated averaged vertical position. The distribution peak is at very small angles $\left(\alpha=0^{\circ}\right)$ and around the mean waterline $(\zeta=0 \mathrm{~m})$, this is indicated by the slope histogram contour lines. The later radiative transfer simulations refer to the framed wave profile of $10 \mathrm{~m}$ length.

\subsection{Determination of wave shadowing effects}

Because of the vertical wave deflections parts of the downwelling insolation from the upper half-space are shadowed (illustrated by the colour labelling in Figure 1). In order to estimate the shadowing, an area with $10 \mathrm{~m}$ (or alternatively unlimited) radius around a wave segment is examined. A subroutine looks for zenith angles of free light incidence, i.e. light beams that are not interrupted by the wave structure. For each wave segment a range of possible light incidence angles is defined, depending on the wave shadowing and its own slope (see Figure 2). Some wave segments can gain additional insolation from multiple wave-reflected light or radiation that is backscattered from the water body and then re-transmitted through the water surface into the direction of the wave segment. The additional light input is probably insignificant; it is not yet regarded in the model. It is further assumed, that there is no feedback from the variance of water-leaving radiation on the isotropic downward directed sky radiation.

Shading of surface parts means that there is neither direct light transmission nor reflection from certain directions, especially 


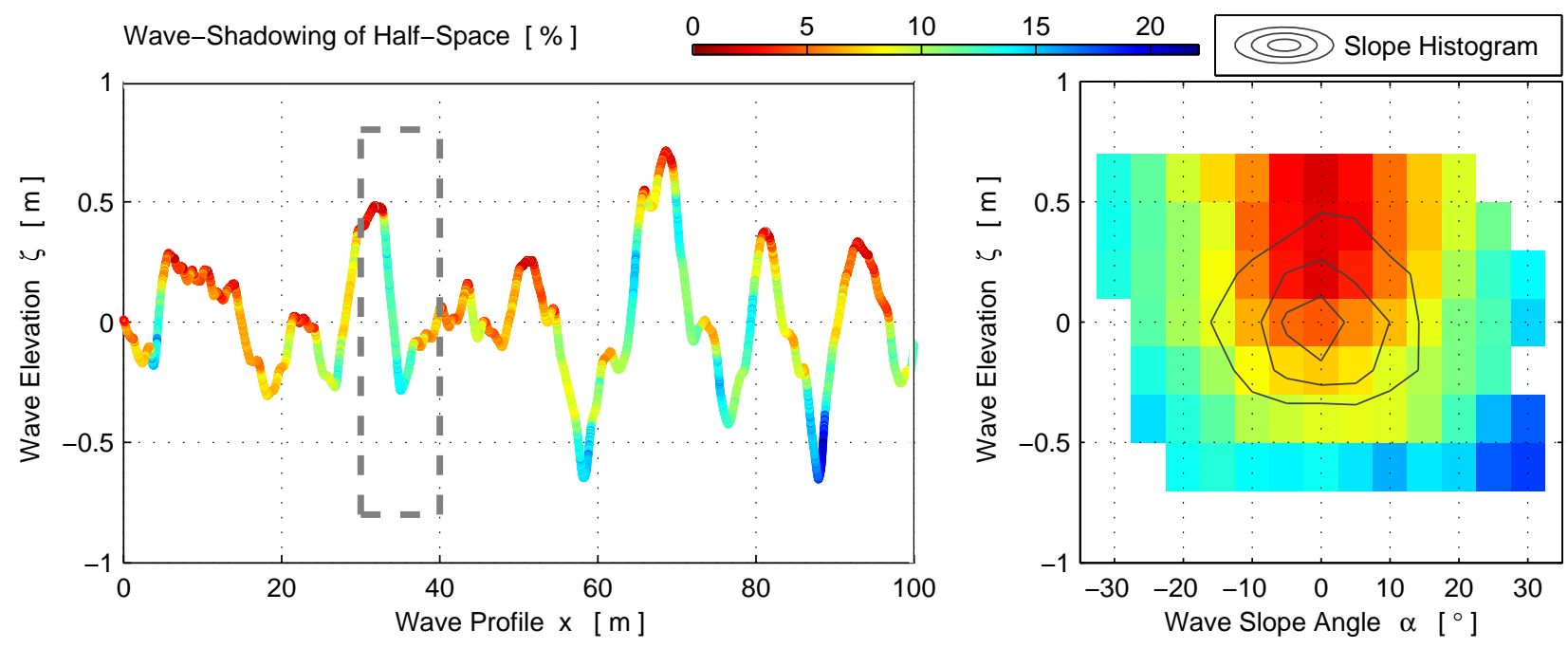

FIG. 1 (Left) Randomly generated two-dimensional wave profile with $\mathrm{H}_{S}=1 \mathrm{~m}, \mathrm{~T}_{p}=4 \mathrm{~S}, \mathrm{U}_{10}=6 \mathrm{~m} \mathrm{~s}^{-1}$, the colour shows the percentage shadowing of the insolation from the upper half-space at any point. (Right) Corresponding distribution of the wave slope angles with mapping of their averaged vertical position and contour lines of the 2-D slope histogram.
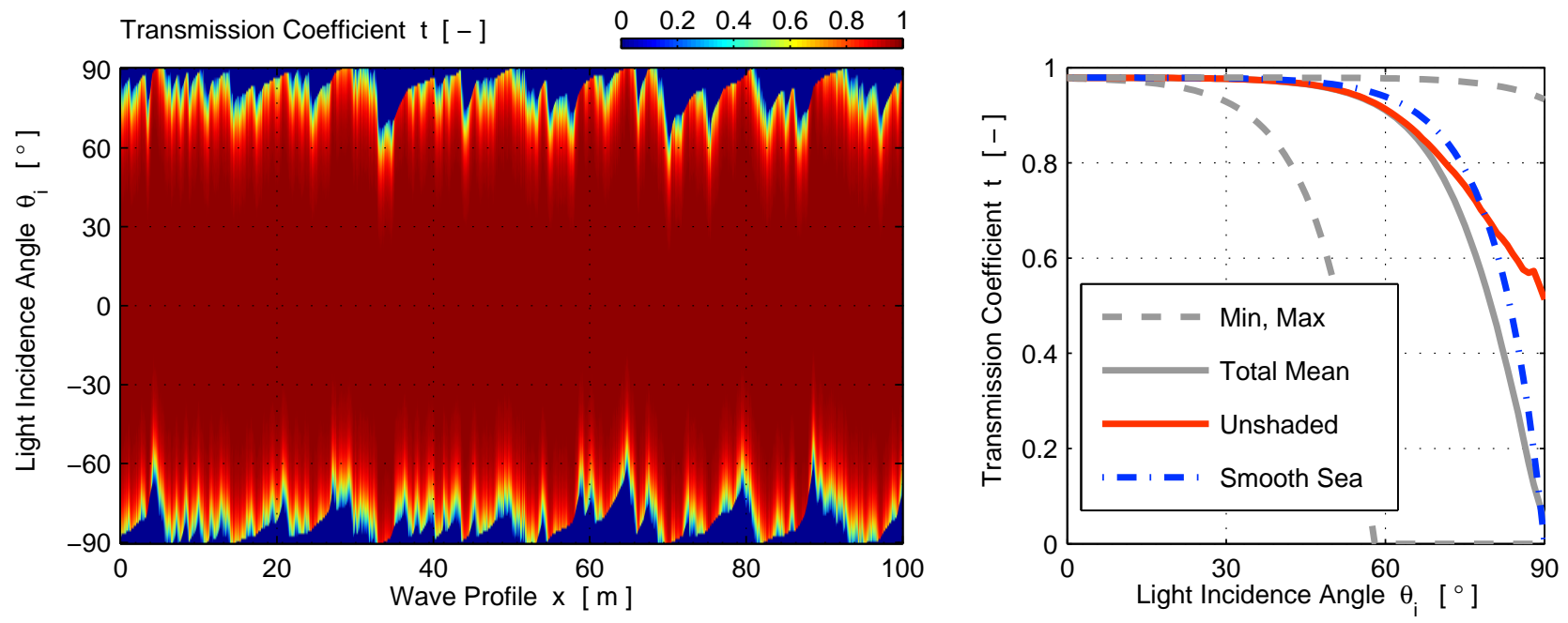

FIG. 2 (Left) Air-to-water transmission coefficient as a function of light incidence angle for every point of the wave profile. (Right) Mean transmission coefficient from the left side (grey solid line) with marking of the minimum and maximum margin of the variability due to the wave structure (dashed lines), mean transmission coefficient of unshaded parts of the wave only (red solid line), and transmission rate for a totally flat surface (dash-dot line).

at incidence angles near the horizon. In order to guarantee always equal light input from all directions, one actually has to reassign the light from shaded parts of the wave to corresponding shading surface segments. But this is not always possible. For example, the area deep down in a wave trough, and thus below the mean water line, is strongly shaded for flat angles (see Figure 1). The corresponding shading surface segment is not clearly assignable. A randomly allocation of the compensated light input at the edge of the model domain would distort the underwater light pattern and thus invalidate the light field statistics. In order to account for this issue, a weighting function is introduced that considers the different surface areas with light input and thus ensures that always the same amount of light input is considered. Figure 3 shows the percentage of the unshaded surface; the reciprocal is the weighting function which is applied to the total underwater light field that results from insolation from a certain direction. At horizontally insolation, $10 \%$ of the total water surface are not shaded, thus $10 \%$ of the photons for the Monte Carlo procedure can be regarded only. Therefore, the resulting light field is amplified by a factor of 10 . This statistical approach, which is worth discussing, is a good proxy of the photon reassignment issue.

\subsection{Program structure and model features}

The flowchart of the computer code differs from the procedure applied by Hieronymi et al. [26]. The new features are described in the following.

In preparation of the actual radiative transfer simulations some databases are created. The first one allocates the twodimensional sea surface in the global space grid (with horizontally $x$ and vertically $z$ coordinates). The second database contains the Fresnel transmission and reflection rates for every wave segment with respect to the incidental light zenith angle 


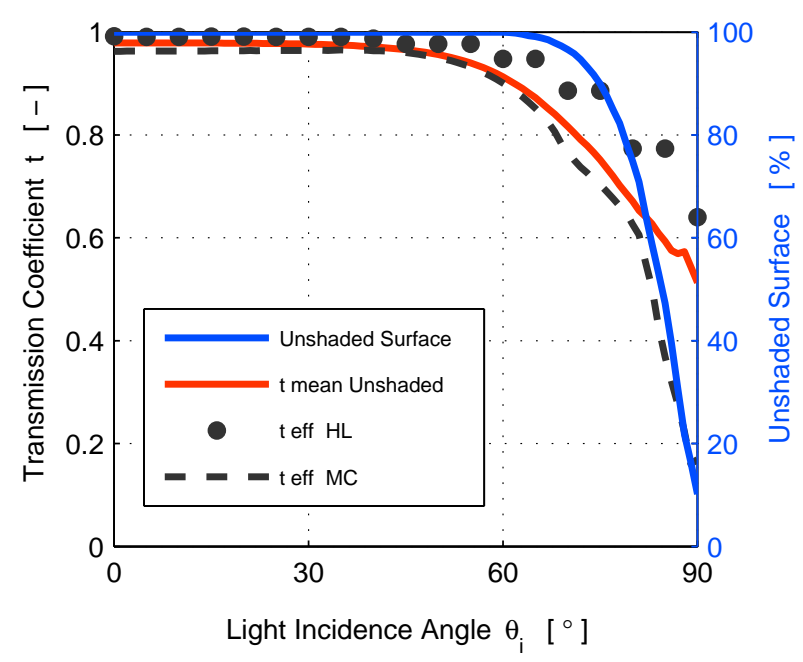

FIG. 3 (Right ordinate) Percentage of the unshaded surface. (Left ordinate) Mean transmission coefficient of unshaded parts of the wave only (red solid line; the same as in Figure 2) and mean effective transmission of the Monte Carlo model (dashed) and of Hydrolight (dots).

$\theta_{i}$, the wave slope angle, and under consideration of wave shadows (Figure 2). This includes both directions from air to sea and reverse. The same is done for transmission and reflection angles according Snell's law. A further database contains the inherent optical properties IOPs of the water body, such as absorption and scattering coefficient and volume scattering function. In theory, the model can work with optically stratified water masses or even with water properties that are assigned to an exact location in the global space grid. However, in this study a well-mixed water body with constant IOPs is assumed.

The simulations are carried out for light incident from every zenith direction of the upper half-space (in $1^{\circ}$ steps, 181 simulations in total). In this manner, it is possible in the end to combine any desired illumination cases, e.g. the percentages of direct sun and diffuse sky radiation, whereby the angular distribution of the sky radiance can be isotropic (which is assumed in this paper) or for example influenced by clouds. In a case of direct sunlight and a clear sky, usually a fraction of $15 \%$ diffuse skylight is assumed [22]. As already mentioned, the insolation from each direction is weighted according to the irradiated surface.

Individual photon packages are released at every wave segment (in this study five photon packages per $0.1 \mathrm{~mm}$ which is up to $5 \times 10^{5}$ per $10 \mathrm{~m}$ wave times 181 light incidence angles). The initial transmission rate and propagation direction is taken from the database. The actual radiative transfer procedure in water follows the Monte Carlo method that is described in Hieronymi et al. [26]. A major difference is the implementation of lateral periodic boundary conditions, as it is used in most other models [13, 22]. If a photon package (or light beam) hits the domain boundaries, its horizontal $x$ coordinate is shifted to the opposite side and the ray-tracing continues. The advantage of periodic boundary conditions is that the photon package remains in the system, its radiant intensity loss can be further traced which saves computa- tional time. The disadvantage is the distortion of the spatial irradiance distribution especially with respect to the wavefocusing effect. A further boundary condition is used for the sea surface. In the case of surface contact, reflection angles and rates are retrieved from the database (thereafter the raytracing procedure continuous inside the water) and the waterto-air transmitted radiance is saved. The lower bound of the simulation domain is actually open; a sea bottom is not intended in this open ocean scenario (the area of interest is down to $40 \mathrm{~m}$ only). The problem of this method is the lack of upwardly directed radiation components at $40 \mathrm{~m}$ depth and therefore an underestimation of upwelling irradiance in the above layer too. For this reason, I limit the validity of the simulation results for upwelling radiation to a depth of approximately $25 \mathrm{~m}$. One cannot expect significant wave-caused upwardly directed irradiance fluctuations at this depth level (which is reconfirmed by the model results). Anyway, at the lower domain boundary (at $40 \mathrm{~m}$ depth) a numerical approximation procedure is called. Depending on the incidence angle, 2.5 to $4 \%$ of all rays are directly backscattered, whereby the values are suggested by Hydrolight.

The entire spatial model domain serves as detector for downand upwelling plane irradiance, $E_{d}(x, z)$ and $E_{u}(x, z)$ respectively (with each 1001 x 821 data points). For statistical analysis purposes, the vertical length of the water column between the actual surface elevation and a detector is defined as reference or true depth $z_{t}$ [26]. Just beneath the water surface, additional detectors for downwelling radiance $L_{d}^{-}(\theta)$ and upwelling radiance $L_{u}^{-}(\theta)$ are built in; the field-of-view of the radiance detectors is $10^{\circ}$. Above the surface, the waterleaving radiance $L_{w}(\theta)$ and upwelling irradiance $E_{u}^{+}$are detected. The downwardly directed radiance distribution above the surface $L_{d}^{+}(\theta)$ is specified by the selected input; the associated surface-reflected radiance from the direct sun and the diffuse sky is derived from $L_{d}^{+}(\theta)$. The total upwelling radiance distribution above water $L_{u}^{+}(\theta)$ can be determined in this way. Thus, the model can provide the total radiation budget at the atmosphere-ocean interface and it can give an estimate on the fluctuations of its individual components.

\section{RESULTS AND DISCUSSION}

\subsection{Benchmark comparison}

First of all, a benchmark comparison with the Hydrolight code is given in order to classify the simulation results of the $\mathrm{MC}$ model. Hydrolight is widely used and has been established as a standard reference model. But we must bear in mind that two completely different approaches are compared. Hydrolight employs invariant imbedding techniques to solve the scalar radiative transfer equation [15]. It is known that this method provides important advantages compared to other solution methods such as discrete-ordinates and Monte Carlo simulations, e.g. it is computationally extremely fast and there is almost no statistical noise caused by the solution method. The main reason for the development of the presented Monte Carlo code is to investigate surface wave effects in more detail.

One point, that can be compared, is the effective light trans- 
mission into the water (see Figure 3). The effective transmission coefficient $t_{\text {eff }}$ is deduced from the ratio of the total downward irradiance just beneath the surface to the value just above, assuming a direct light source only (i.e. black sky). There is a good agreement for high standing sun, i.e. incident light with zenith angles up to approximately $45^{\circ}$ (this is half of the upper half-space). In the MC model, the light transmission into water approaches 10 to $20 \%$ for angles near the horizon; most of the light is then reflected at the surface. Uncertainties of these values may arise from the step-line registration of $E_{d}$ below the surface. The effective transmission rate of the MC model lies between the total mean value of the Fresnel transmission (including zero-transmission for shaded parts) and the mean of the only-unshaded wave segments (Figure 2, right). In every case, the effective transmission rate from Hydrolight is significantly higher for light incidence angles near the horizon; although, the course of the curve fits to the wind-related irradiance reflectance-considerations of Preisendorfer and Mobley [30]. But we should keep in mind that, according to Mobley [20], Hydrolight cannot properly simulate the radiative transfer across wind-blown surfaces for solar zenith angles that are very near the horizon.

The irradiance decrease in the water column is compared for different solar zenith angles $\theta_{s}$ in case of a clear sky (with $15 \%$ diffuse sky radiation) and the ratio of diffuse to direct light source where the sun is at $\theta_{s}=0^{\circ}$. Figure 4 shows the percentage deviation

$$
\varepsilon=100 \frac{\overline{E_{M C}}-\overline{E_{H L}}}{\overline{E_{H L}}}
$$

(unit [\%]) of the computed mean irradiances for the downwardly (upper images) and upwardly (below) directed irradiances, $\overline{E_{d}}$ and $\overline{E_{u}}$ respectively (MC for the Monte Carlo model, $\mathrm{HL}$ for Hydrolight). In computations of $E_{d}$ and $E_{u}$, comparisons of numerical models usually give deviations within a few percent of each other throughout the euphotic zone, and the error of in-situ measurements is typical lower than $5 \%$ $[19,31]$. The white colour in Figure 4 indicates a deviation of less than $\pm 5 \%$, red colours show an overestimation of the MC model of more than $5 \%$, and blue is for an underestimation respectively. With regards to $E_{d}\left(\theta_{s}\right)$, there is a relatively good agreement in the upper water layer up to solar zenith angles of $60^{\circ}$. At angles near the horizon, MC results in up to $70 \%$ less downwelling irradiance compared to HL; this is probably largely due to the fact that significantly less radiation is transmitted into the water. At higher sun angles and water depths of more than $20 \mathrm{~m}$, MC overestimates $E_{d}\left(\theta_{S}\right)$ with up to $7 \%$. With regard to the downwelling irradiance as function of diffuse sky, the maximum deviation is in the order of $8 \%$. The upwelling irradiance (and also radiance) is subject to substantial Monte Carlo statistical fluctuations and in any case is clearly underestimated by the MC model. $E_{u}\left(\theta_{s}\right)$ is underestimated by 8 to $28 \%$ for zenith angles that are not near the horizon and by almost $80 \%$ for sun insolation from $90^{\circ}$. The $E_{u}$ dependency of the daylight diffuseness shows differences mostly in the order of 10 to $20 \%$. These differences, in particular regarding $E_{u}$, cannot be denied and future efforts will focus on model validation with in-situ measurements, also with respect to the simulated light field variability. Generally, the model precision can be improved by increasing the number of traced photon packages which was not feasible for this

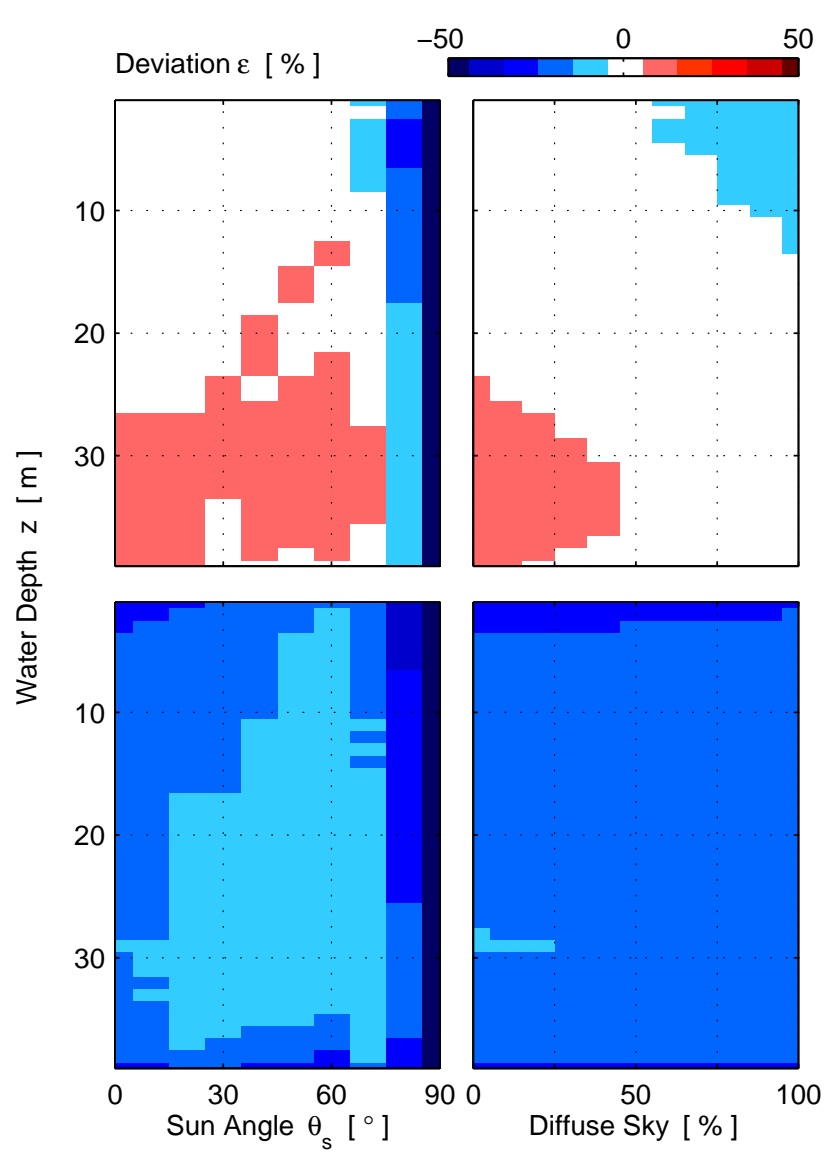

FIC. 4 Relative difference between the results of the Monte Carlo model and Hydrolight. (Upper panels) Averaged downwelling irradiance as function of solar zenith angle at clear sky (left) and as function of fraction of diffuse skylight with the sun at zenith (right). (Lower row) Corresponding upwelling irradiance.

study. However, the differences shown here are still comparable with those of other benchmarking studies [13, 17, 19, 22]).

\subsection{Mean and variance of light transmission}

The general influence of a wind-roughened sea surface and the light incident angle on the average irradiance transmission through the air-sea interface has long been known $[5,30]$. The exact description of the wave surfaces, including vertical wave deflections, allows an estimation of the wave shadowing effect, its influence on the mean transmission rate, and a statement on the variability of the transmission.

As already mentioned (Section 2.2), Figure 1 shows the utilized wave profile with colour-indicated shadowing of the upper half-space at any point together with the distribution of wave slopes and their vertical position. Only the peaks of high wave crests gain undisturbed light from the entire halfspace. The wave slope angles are small (around $0^{\circ}$ ) at the wave peaks. Hence, the light transmission is also close to zero for high zenith angles. In wave troughs strongest shadowing effects occur; the light input with flat incidence angles near the horizon is blocked by the wave structure. In this example up to $25 \%$ of the half-space are shadowed. Also the wave slope itself causes shadowing. The deeper a wave segment lies and 
the steeper its slope is, the stronger is the shadowing of the incoming light. This implies that wave shadowing depends on the steepness of the prevailing large gravity waves (i.e. on the sea state). Locally limited wind generates capillary and small gravity waves, but no serious vertical wave deflections. Short high gravity waves of a wind sea shadow more incoming light than long swell waves.

It is clear that the wave shadowing affects light with high incidence angle $\theta_{i}$ (near the horizon) only. Figure 2 illustrates the angular dependency of the transmission coefficient $t$. In case of perpendicular singular light input (i.e. $\theta_{i}=0^{\circ}$, black sky), the transmission rate varies between 97.74 and $97.89 \%$. The coefficient of variation

$$
C V=100 \frac{\sigma}{\mu}
$$

(unit [\%]), which is the percentage ratio of standard deviation $\sigma$ to the mean value $\mu$, is $0.0087 \%$. Indeed, for light incidence angles between $0^{\circ}$ and $45^{\circ}$ (i.e. high solar altitudes), $C V$ never exceeds $2 \%$. Thus, the variance of the transmission due to wind-waves is negligibly small at high sun; roughly $97 \%$ of the light is transmitted. That means that the transmittance is actually wind-independent for solar zenith angles (and surface viewing angles) $<45^{\circ}$; the light transmission is the same as the Fresnel transmittance for a flat surface (dotdashed line), which is also discussed by Preisendorfer and Mobley [30] and Gordon [21]. Whereas at a horizontal light incidence $\left(\theta_{i}=90^{\circ}\right)$, the strongest fluctuations of the transmission rate occur; the values vary between 0 and $95 \%(\mathrm{CV}=$ $330 \%$ ) but the total light transmission (solid grey line) is below $5 \%$, because $90 \%$ of the wave structure is self-shaded without any light transmission, i.e. at these points actually no reflection occurs too. The red solid line shows the mean transmission coefficient of the $10 \%$ unshaded wave; at $\theta_{i}=$ $90^{\circ}$, the average transmission is approximately $50 \%$. One can argue that these parts of the wave shade the entire rest and that steeper wave slopes, with corresponding higher transmission rates, shade larger areas. Effectively, there is less than $20 \%$ transmitted light when comparing the angle-weighted downwelling irradiance above and below the surface in the MC model (already mentioned, see Figure 3 ). For $\theta_{i}$ between $50^{\circ}$ and $80^{\circ}$, the courses of the mean transmission coefficients (both solid lines in Figure 2) are underneath the dot-dashed line for a flat surface (wind speed of $0 \mathrm{~m} \mathrm{~s}^{-1}$ ). This relationship and the much lower transmittance at horizontal light incidence are in contrast to the significantly higher values that are assessed by Preisendorfer and Mobley [30] and Gordon [21] and that are applied in Hydrolight (discussed in the above Section 3.1; note that the assumed wind speed is $6 \mathrm{~m} \mathrm{~s}^{-1}$ ). This leads to my assumption that due to the wave shadowing the light transmission into water up to now is clearly overestimated for low solar altitudes (i.e. high $\theta_{i}$ ).

The wave shadowing also affects the subsurface upwardly directed radiance. Near beneath a wave crest the radiance from flat incidence angles (i.e. high $\theta_{i}$ ) is reduced which leads to a reduction of the total upwelling irradiance in this area. This is slightly visible in Figure 5, where the spatial distribution of $E_{u}$ is shown for a case with total isotropic insolation.
With regards to the water-to-air transmission such effects play a minor role because Snell's law allows transmission for low incidence angles $\theta_{i}$ between 0 and $48^{\circ}$ only (Snell's window); light is totally reflected at larger angles.

\subsection{Mean and variance of the irradiance in water}

Examples of the simulated spatial irradiance distributions along the water column are shown in Figure 5. The upper row shows the downwelling plane irradiance $E_{d}$, the upwelling plane irradiance $E_{u}$ is beneath, and the lowermost row shows the irradiance reflectance $R$ which is the ratio of upwelling to downwelling plane irradiance. Three cases are considered (from left to right): Sun at zenith in a clear sky, sun at $\theta_{s}=60^{\circ}$ in a clear sky, and $100 \%$ diffuse isotropic irradiation. The related statistical summary, regarding mean values and coefficients of variation, is presented in Figure 6. The change of both parameters is shown for clear sky with $15 \%$ diffuse skylight and different solar zenith angles (the both columns left) and for different fractions of diffuse to direct light whereas the sun is at $\theta_{S}=0^{\circ}$ (both right columns).

\subsubsection{Influence of the solar zenith angle}

The left column of Figure 6 depicts the mean irradiances and their ratio in a diurnal cycle. The values are always related to $100 \%$ of the surface downward irradiance. $E_{d}$ enters deepest into the water column at high solar altitudes, but rapidly diminishes for sun positions towards the horizon. $E_{u}$ reaches highest values at $\theta_{S}$ between 60 and $70^{\circ}$. Light in natural water is scattered mostly into forward directions; a light beam develops a spread field around it and the light is increasingly diffuse [26]. If the spread field is strongly inclined due to shallow insolation, proportionately more radiation is upwardly directed and $E_{u}$ increases. The irradiance reflectance $R$ is considered as an apparent optical property AOP of the water body. The change of the irradiance ratio with depth is rather small, but there is a gradient along the water column. However, we see significant changes in $R$ as the sun zenith angles changes; differences can be in the order of $50 \%$.

The higher the sun is, the deeper reaches the light variability. $\mathrm{CV}$ is a measure for the magnitude of radiative enhancements relative to the mean value which changes with solar position. Additionally, the focusing-effectiveness of differently long waves at the surface [11] changes too, e.g. at $10 \mathrm{~m}$ depth, the fluctuation frequency of $E_{d}$ is more influenced by small scale waves (high frequency) at $\theta_{s}=0^{\circ}$ compared to $60^{\circ}$ insolation, where the fluctuations depend more on larger gravity waves (see Figure 5). Both aspects cause higher $C V$ values at $10 \mathrm{~m}$ depth for e.g. $45^{\circ}$ insolation compared to $0^{\circ}$ (Figure 6(b)). Directly beneath the surface within the first centimetres, $E_{d}$ variability is small because the lensing effect of waves initially needs to build up [11]. At high sun altitudes the maximum light variability is located slightly deeper (at approximately $1 \mathrm{~m}$ depth, here $C V$ of $E_{d}\left(\theta_{s}=0^{\circ}\right)$ is $44 \%$ ) compared to cases with low sun altitudes (roughly 10 to $80 \mathrm{~cm}, C V\left(\theta_{S}=85^{\circ}\right.$ ) is $100 \%)$. The wave shadowing effect and the corresponding selective light beam input originate the high $C V$ values. This ob- 

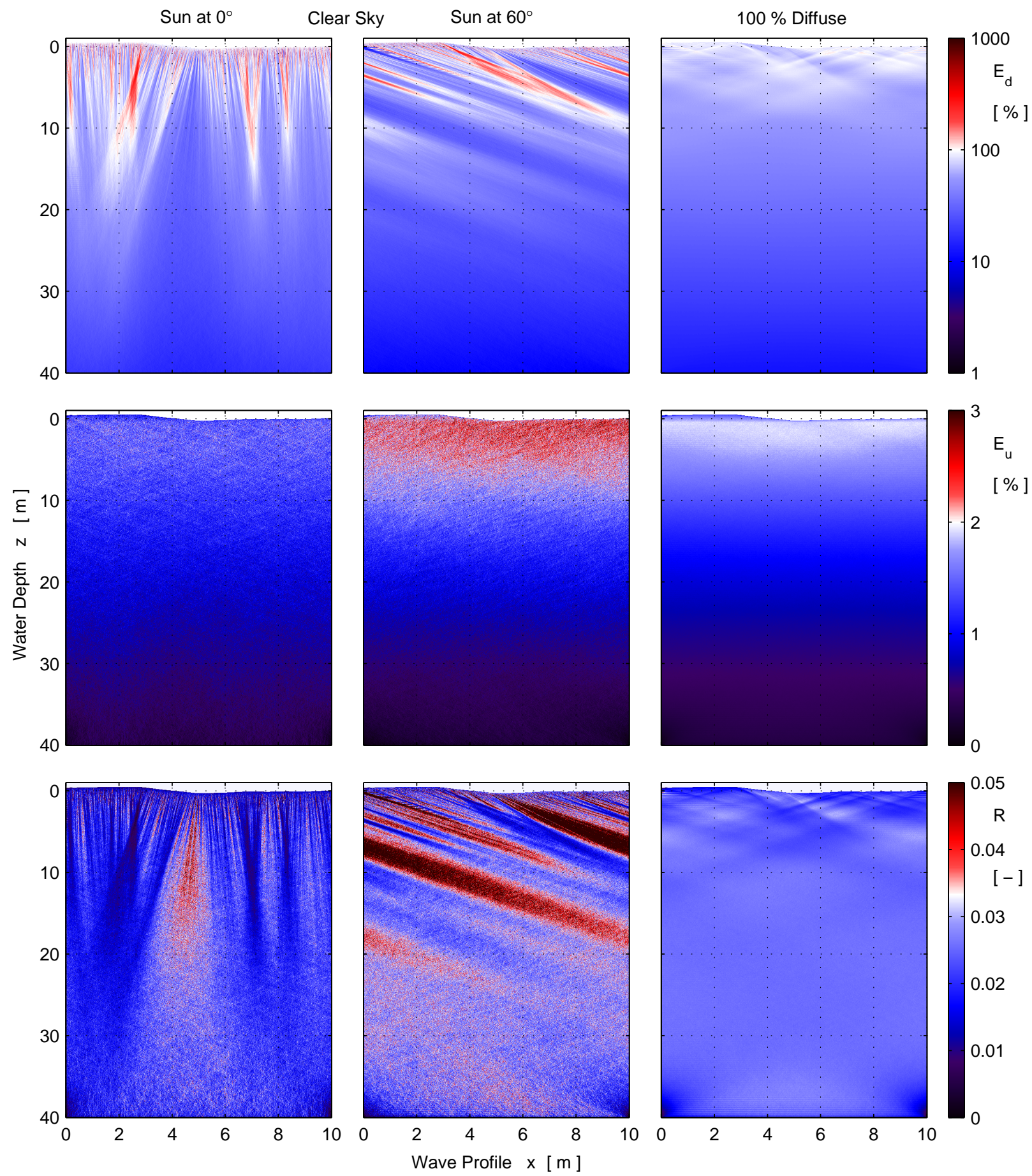

FIG. 5 Spatial distribution of the underwater downwelling irradiance $E_{d}$ (upper panels, with logarithmic colour scale), upwelling irradiance $E_{u}$ (middle row), and irradiance reflectance $R$; the both left columns show clear sky cases with a fraction of $15 \%$ diffuse sky radiation for perpendicular sun insolation and a sun zenith angle of $60^{\circ}$; the right column shows an case with completely isotropic insolation (100\% diffuse sky radiation).

servation relativizes the previous assumption that light fluctuations are most effective with high sun altitudes.

$C V$ of $E_{u}$ is relatively homogeneous at given depths and for solar zenith angles that are not near the horizon. For high zenith angles, $C V$ again is dominated by the wave shading which leads to strong variability. However, variance of $E_{u}$ due to wave focusing can be slightly recognized, but the results are strongly affected by Monte Carlo noise, especially for cases with clear skies.
$C V$ of $R$ is mainly determined by fluctuations of $E_{d}$. Thus, the light variability with respect to $E_{d}$ or $R$ is relatively small at high sun altitude and very close to the surface, or below the top water layer of about $10 \mathrm{~m}$.

\subsubsection{Influence of the daylight diffuseness}

The mean downwelling irradiance and the lensing efficiency of waves are clearly reduced if we increase the diffuse sky radiation (Figure 6(c),(d)) which fits to observations 

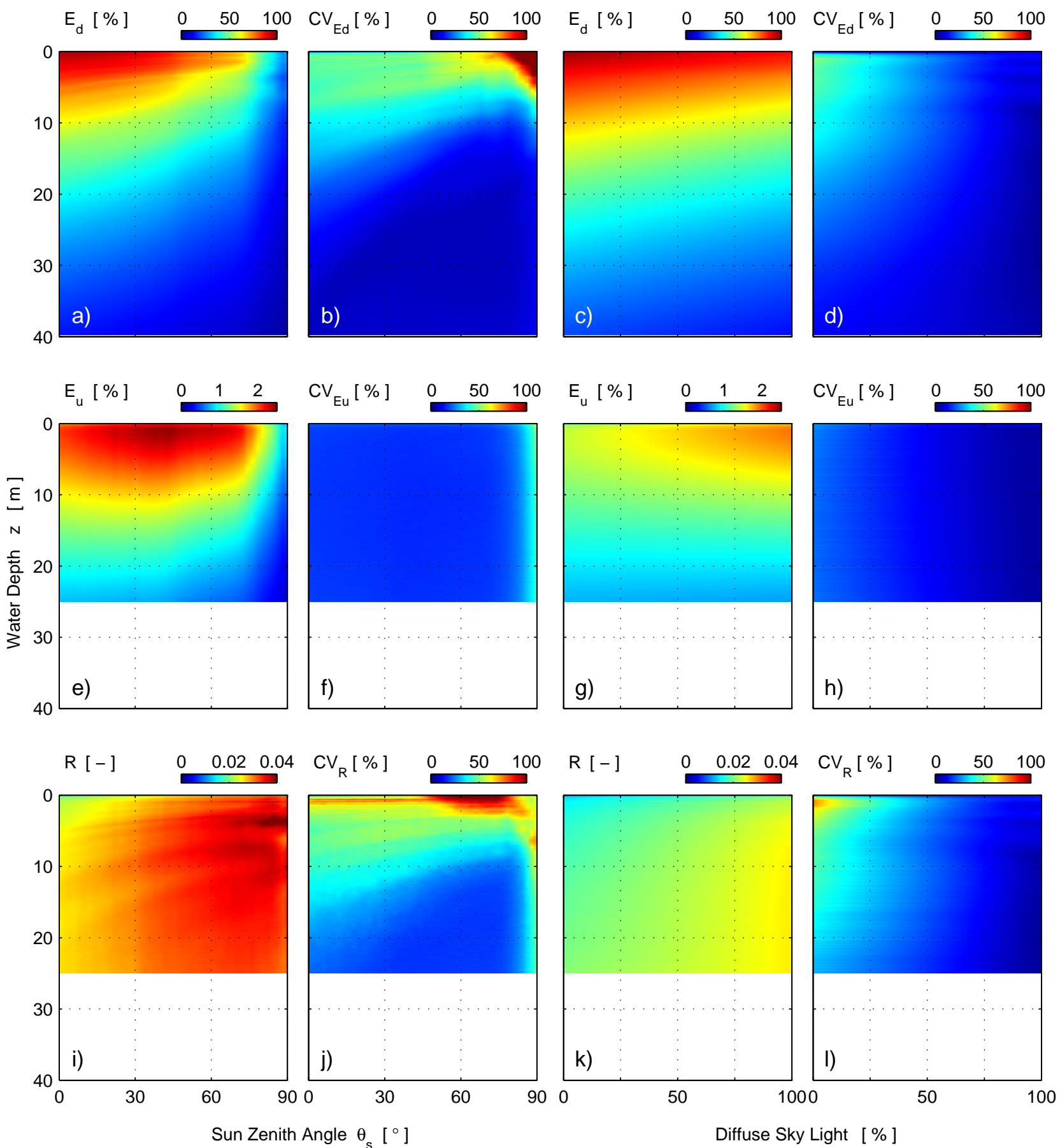

FIG. 6 Underwater light field statistics as function of the solar zenith angle at clear sky with $15 \%$ diffuse skylight (the both left columns) and as function of the fraction of the diffuse sky radiation whereas the sun is in zenith (both right columns). (First row) Downwelling irradiance, (second row) upwelling irradiance, and (lower row) irradiance reflectance, in each case the spatially averaged values are shown with the respective coefficient of variations.

$[4,6,7]$. Gernez and Antoine [7] also documented the associated change of the temporal fluctuation rate and stated that the average fluctuation period is approximately half of the period of the dominant surface wave, whereas Hieronymi and Macke [12] gave an explanation of the reasons therefor. This is mainly due to the conical shape of the underwater irradiance distribution which is caused by refraction at the surface and incidence-weighted transmission and the resulting stronger influence of larger gravity waves at the surface. The upper row of Figure 5 illustrates the relationship. At clear sky, the variability of $E_{d}$ in the top layer is governed by high-frequency changes that are associated with wind-related capillary and small gravity waves at the surface [11]. In case of an overcast sky, the fluctuation rate is strongly reduced and rather dependent on larger surface waves.

With regards to the $E_{u}$, the increase of $E_{u}$ towards increasing sky diffuseness (Figure 6(g)) can be explained by means of Figure 6(e), where the angular dependency of $E_{u}$ is shown. Shallow angles gain more and more influence and thus pro- 
portionately more light is upwardly directed. $C V$ of $E_{u}$ shows a stronger dependency on the daylight diffuseness than on solar zenith angles (Figure 6(h)), although a part of the difference is due to Monte Carlo statistical fluctuations which are reduced for increasing diffuseness. However, the image in the right column in the centre of Figure 5 adumbrates the influence of the wave shadowing on $E_{u}$ in the area near beneath the wave crest (on the left side).

The irradiance reflectance has a similar dependency as with the sun position, but much weaker. There is a slightly increase of $R$ towards increasing diffuseness (Figure $6(\mathrm{k})$ ). $C V$ of $R$ shows high variability at very low portion of diffuse sky light (Figure 6(1)). Thus, the light variability with respect to $E_{d}$ or $R$ is relatively small in cases with stronger weighting of the sky radiation and very close to the surface, or below the top water layer of about $10 \mathrm{~m}$.

\subsubsection{Underwater light flashes}

Generally, high solar altitudes and clear skies enable the light to penetrate deeply into the water and consequently cause deep-reaching variability. Underwater light flashes, which are according to Dera and Stramski [3] defined as downwelling irradiance pulses that exceed the mean irradiance by a factor of more than 1.5, can reach water depths of approximately $25 \mathrm{~m}$ for perpendicular solar insolation in a clear sky, but $12 \mathrm{~m}$ for $\theta_{s}=60^{\circ}$ only (note that the model resolution and irradiance detector size influence the depth-prediction of light flashes $[1,26])$. The highest $E_{d}$ peaks are mostly in the order of the four-fold of the mean irradiance at their respective depth. The depth of the maximum radiative enhancement depends on the solar zenith angle; the maximum comes closer to the surface with lower sun altitudes. Figure 6(b) indicates an extreme variability of $E_{d}$ near the surface for solar zenith angles near the horizon. Here we have indeed highest irradiance peaks, but this is rather related to the wave shadowing of large areas and the massively reduced mean irradiance under water. Anyway, there is still a wave lensing effect at the illuminated side of the wave crests and the related $E_{d}$ enhancements compared to the mean are the strongest at all. There are no light flashes for the case with an overcast sky (100\% diffuse); the maximum downwelling irradiance enhancement is in the order of $25 \%$ near the surface. Furthermore, no light flashes were observed for the upwelling irradiance.

\subsection{Mean and variance of the upwelling radiance just below the surface}

In order to underline the angular dependency of $E_{u}$ and thereby $R$, the averaged upwelling radiance distribution just below the surface $L_{u}^{-}(\theta)$ is shown for the clear sky case as a function of sun zenith angle, together with the corresponding coefficient of variation (Figure 7$)$. At vertical irradiation $\left(\theta_{s}=0^{\circ}\right)$, there is a local minimum of $L_{u}^{-}(\theta)$ in the direct backscatter direction $\left(\theta=0^{\circ}\right)$ and two similar maxima at $\theta$ $= \pm 60^{\circ}$. For a flat surface, we would expect the maximum in the direct backscatter direction. But due to the wind-blown surface with many inclined spread light beams, there is an additional gain into lateral directions of the upwelling radiance. At relatively shallow sun incident between 40 and $85^{\circ}$, we observe strongest upwelling radiance values. The coefficient of variation with respect to $L_{u}^{-}(\theta)$ mainly indicates the large variability in cases with small radiance values.

The image of the right hand side of Figure 7 refers to simulations with a black sky. It shows the percentage of photons packages that are backscattered in the water column and hit the lower side of the water surface (solid line). A part of the photons is completely reflected at the surface (dashed line). About twice as many photon packages reach the surface at a light incidence angle $\theta_{i}$ of $70^{\circ}$ in comparison with perpendicular incidence. The ratio of total reflection (red line) increases a little with increasing $\theta_{i}$, namely from 43 to $47 \%$. For those reasons, we see a corresponding angular dependency of the water-leaving radiance too.

\section{CONCLUSIONS}

\subsection{Classification of the findings}

Field measurements and numerical studies have shown that maximum downward irradiance fluctuations near the surface arise at low wind speeds up to $5 \mathrm{~m} \mathrm{~s}^{-1}[3,7,10,11]$ and that strongest intensity fluctuations below the upper water layers $(>10 \mathrm{~m})$ are caused by larger gravity waves in a sea state $[2,8,11]$. It is known that the intensity of light fluctuations drops rapidly at higher wind speeds, which is caused by a suite of phenomena associated with very effective scattering of direct sunlight by the increasing surface roughness and the presence of foam and underwater bubble clouds [5, 32]. This study refers to frequently occurring sea characteristics with $1 \mathrm{~m}$ wave height and a prevailing wind speed of $6 \mathrm{~m} \mathrm{~s}^{-1}$ and white caps and bubbles are ignored; from this point of view, very effective wave-focusing can be expected.

The influence of the solar zenith angle on $E_{d}$ variability has been measured for $\theta_{s}>20^{\circ}$ within the upper metres $[7,10]$, whereby a clear decrease of $C V$ towards high angles was noticed. In general, this trend is confirmed by this theoretical investigation, but the behaviour is actually much more differentiated. For example, at $10 \mathrm{~m}$ depth, $C V$ of $E_{d}$ has a maximum at sun angles between 30 and $60^{\circ}$; at higher sun altitudes stronger irradiance enhancements, i.e. light flashes, can be observed, but $C V$, which depends on the mean value, is slightly reduced (Figure 6(b)). And very close to the surface, within the first tens of centimetres, $C V$ increases with lower sun elevations. The theoretical maximum of $C V$ is at incidence angles near the horizon, because large areas of the wave system are shadowed and hence the mean value of $E_{d}$ is sharply reduced.

With regards to the effect of daylight diffuseness, the model basically confirms previous results $[4,6]$. The focusing of sunlight is most effective under clear skies. $C V$ is significantly smaller under overcast conditions. The corresponding drastic decrease in frequency and maximum intensity of underwater flashes can be explained by means of the model.

There are other factors that influence the underwater light variability, but they are not considered in this study. For 

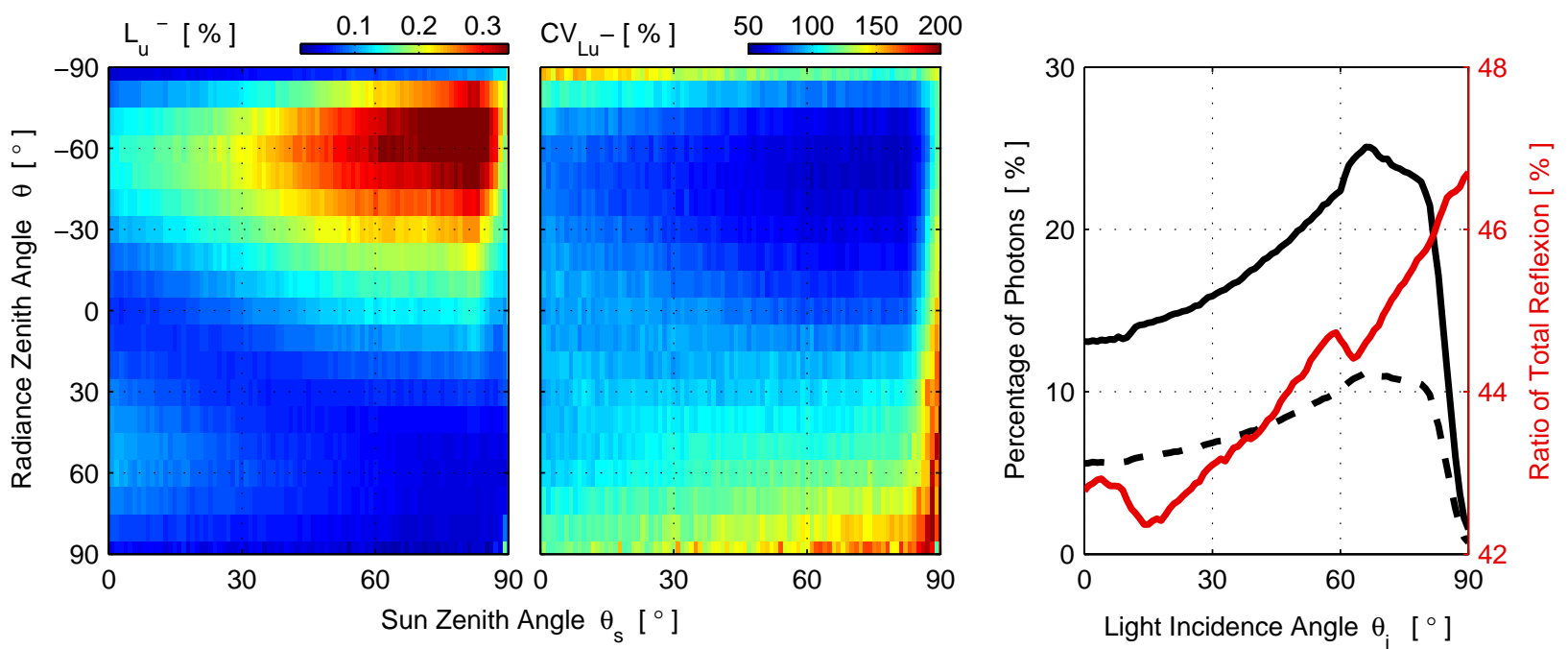

FIG. 7 (Left) Upwelling radiance $L_{u}^{-}(\theta)$ just below the surface at clear sky (15\% diffuse skylight) as function of the sun position. (Central panel) Corresponding coefficient of variations of the upwelling radiance. (Right) Monte Carlo simulations of the percentage upwardly-directed surface contact of individual photons as function of their initial incidence angle (black solid line), the dashed line marks the fraction that undergoes total reflection in water, and the red line shows the corresponding ratio of both.

example, irradiance variability depends on the wavelength of light too. At a reference depth in the top layer, $C V$ is higher in the yellow-red waveband compared to the greenblue band which is considered in this paper [1, 6, 7, 9]. Furthermore, properties of the boundary layer between atmosphere and water affect the radiative transport and hence the variability. This includes for example salinity and temperature effects [13] or surface micro-layers. Also an ice layer can significantly enhance the underwater downwelling irradiance, which is mainly due to light in the ice that is scattered upwardly and then totally reflected by the air-ice interface [33].

\subsection{Implications for radiometric measurements}

It is known that optical profiles in water, especially regarding $E_{d}$, show large uncertainties due to wave focusing effects [31]. This applies to all modern sensor deployment platforms as for example free-fall profilers, surface floats, and multiple sensor units that are placed at different vertical depths on moorings [34]. If one wants to measure $E_{d}$ in the upper water layer, most intense fluctuations and therefore large measuring inaccuracy must be expected. As a result of the presented theoretical considerations, it can be advisable to measure $E_{d}$ and $E_{u}$ very close to the surface by means of a surface float, i.e. rather in $10 \mathrm{~cm}$ depth than underneath $50 \mathrm{~cm}$, because here the lensing effect of small waves has not yet fully developed. In addition, it is better to measure at noon with high sun altitudes. One must, on the other hand, be mindful of the fact that hydrodynamic disturbances of the measurements can occur that change the sensor tilt.

It has been shown that the average irradiance reflectance, which is considered an apparent optical property of the water body, depends on the solar zenith angle but significantly less on changes of the daylight diffuseness. Future in-situ measurement campaigns should investigate the dependency of the irradiance reflectance from sun zenith angles changes as well as the practicality of $E_{d}$ measurements very near the surface.

\subsection{Implications for radiative transfer modelling}

The influence of surface roughness is small for a wide range of sun-viewing geometries and the transmittance of the airwater interface is nearly identical to that for a flat surface [21]. This is confirmed for low sun zenith angles $<45^{\circ}$. However, wave shadowing by large gravity waves become increasingly important for sun directions near the horizon. At shadowed parts of the surface, neither transmission nor reflection of direct sunlight occurs. The effective transmission of light depends on the wave structure, i.e. it is sea state-depending, and is much smaller than expected. This leads to my assumption that due to the wave shadowing the light transmission into water up to now is clearly overestimated for low solar altitudes (i.e. high $\theta_{i}$ ). Further investigations on wave shadowing effects on the transmittance and reflectance should be objective of future works.

\section{ACKNOWLED GEMENTS}

The author benefited from conversations with Andreas Macke, Ullrich Hieronymi, Dagmar Müller, Hajo Krasemann, and Rüdiger Röttgers. My thanks also go to the anonymous reviewer for helpful comments and suggestions.

\section{References}

[1] M. Darecki, D. Stramski, and M. Sokolski, "Measurements of highfrequency light fluctuations induced by sea surface waves with an underwater porcupine radiometer system," J. Geophys. Res. 116, CoOHog (2011). 
[2] V. P. Nikolayev, and V. G. Yakubenko, "On the relationship between the statistical characteristics of the underwater light field and the wave state characteristics," Izv. Atmos. Ocean. Phy. 14(1), 88-92 (1978).

[3] J. Dera, and D. Stramski, "Maximum effects of sunlight focusing under a wind-disturbed sea surface," Oceanologia 23, 15-42 (1986).

[4] D. Stramski, "The effect of daylight diffuseness on the focusing of sunlight by sea surface waves," Oceanologia 24, 11-27 (1986).

[5] R. E. Walker, Marine light field statistics (Wiley Series in Pure and Applied Optics, New Jersey, 1994).

[6] M. Stramska, and T. Dickey, "Short-term variability of the underwater light field in the oligotrophic ocean in response to surface waves and clouds," Deep-Sea Res. I 45, 1393-1410 (1998).

[7] P. Gernez, and D. Antoine, "Field characterization of wave-induced underwater light field fluctuations," J. Geophys. Res. 114, C06025 (2009).

[8] M. Hieronymi, and A. Macke, "Spatiotemporal underwater light field fluctuations in the open ocean," J. Europ. Opt. Soc. Rap. Public. 5, 10019S, 1-8 (2010).

[9] P. Gege, and N. Pinnel, "Sources of variance of downwelling irradiance in water," Appl. 0pt. 50(15), 2192-2203 (2011).

[10] P. Gernez, D. Stramski, and M. Darecki, "Vertical changes in the probability distribution of downward irradiance within the nearsurface ocean under sunny conditions," J. Geophys. Res. 116, CoOHo7 (2011).

[11] M. Hieronymi, and A. Macke, "On the influence of wind and waves on underwater irradiance fluctuations," Ocean Sci. 8, 455-471 (2012).

[12] M. Hieronymi, and A. Macke, "Variability of downwelling irradiance in the upper ocean in response to surface waves and diffuse sky radiation," in Proceedings of $27^{\text {st }}$ Ocean Optics Conference 2012, (Glasgow, 2012).

[13] Z. Xu, X. Guo, L. Shen, and D. K. P. Yue, "Radiative transfer in the ocean turbulence and its effect on the underwater light field," J. Ceophys. Res. 117, $\mathrm{CoOH} 18$ (2012).

[14] C. Cox, and W. Munk, "Measurements of the roughness of the sea surface from photographs of the sun's glitter," J. Opt. Soc. Am. 44(11), 838-850 (1954).

[15] C. D. Mobley, "Light and water: Radiative transfer in natural waters," (Academic Press, San Diego, 1994).

[16] F. Fell, and J. Fischer, "Numerical simulation of the light field in the atmosphere-ocean system using the matrix-operator method," J. Quant. Spectrosc. Ra. 69, 351-388 (2001)

[17] K. I. Gjerstad, J. J. Stamnes, B. Hamre, J. K. Lotsberg, B. Yan, and K. Stamnes, "Monte Carlo and discrete-ordinate simulations of irradiances in the coupled atmosphere-ocean system," Appl. 0pt. 42(15), 2609-2622 (2003).

[18] A. Rozanov, V. Rozanov, M. Buchwitz, A. Kokhanovsky, and J. P. Burrows, "SCIATRAN 2.0 - A new radiative transfer model for geophysical applications in the 175-2400 nm spectral region," Adv. Space. Res. 36(5), 1015-1019 (2005).
[19] C. D. Mobley, B. Gentili, H. R. Gordon, Z. Jin, G. W. Kattawar, A. Morel, P. Reinersman, et al., "Comparison of numerical models for computing underwater light fields," Appl. 0pt. 32(36), 74847504 (1993).

[20] C. D. Mobley, "How well does Hydrolight simulate wind-blown sea surfaces?," Hydrolight Technical Note 1, http://www.sequoiasci. com/products/hl-radiative.cmsx (2002).

[21] H. R. Gordon, "Normalized water-leaving radiance: revisiting the influence of surface roughness," Appl. 0pt. 44(2), 241-248 (2005).

[22] D. D'Alimonte, G. Zibordi, T. Kajiyama, and J. C. Cunha, "Monte Carlo code for high spatial resolution ocean color estimation," Appl. Opt. 49(26), 4936-4950 (2010).

[23] J. R. V. Zaneveld, E. Boss, and P. A. Hwang, "The influence of coherent waves on the remotely sensed reflectance," Opt. Express 9(6), 260-266 (2001).

[24] Y. You, D. Stramski, M. Darecki, and G. W. Kattawar, "Modeling of wave-induced irradiance fluctuations at near-surface depths in the ocean: a comparison with measurements," Appl. Opt. 49(6), 1041-1053 (2010).

[25] S. Kay, J. Hedley, S. Lavender, and A. Nimmo-Smith, "Light transfer at the ocean surface modeled using high resolution sea surface realizations," Opt. Express 19(7), 6493-6504 (2011).

[26] M. Hieronymi, A. Macke, and 0. Zielinski, "Modeling of waveinduced irradiance variability in the upper ocean mixed layer," Ocean Sci. 8, 103-120 (2012).

[27] M. Hieronymi, and A. Macke, "Wave-induced irradiance variability in the upper ocean from modeling and observations," AIP Conf. Proc. 1531, 915-918 (2013).

[28] M. Wang, K. D. Knobelspiesse, and C. R. McClain, "Study of the Sea-Viewing Wide Field-of-View Sensor (SeaWiFS) aerosol optical property data over ocean in combination with the ocean color products," J. Geophys. Res. 110, D10S06 (2005).

[29] T. J. Petzold, "Volume scattering functions for selected ocean waters," SIO Ref. 72-78 (Scripps Institution of Oceanography, San Diego, 1972).

[30] R. W. Preisendorfer, and C. D. Mobley, "Albedos and glitter patterns of a wind-roughened sea surface," J. Phys. Oceanogr. 16(7), 1293-1316 (1986).

[31] G. Zibordi, D. D'Alimonte, and J. F. Berthon, "An evaluation of depth resolution requirements for optical profiling in coastal waters," J. Atmos. Ocean. Tech. 21(7), 1059-1073 (2004).

[32] D. Stramski, and J. Tegowski, "Effects of intermitted entrainment of air bubbles by breaking wind waves on ocean reflectance and underwater light field," J. Ceophys. Res. 106, 31345-31360 (2001).

[33] S. Jiang, K. Stamnes, W. Li, and B. Hamre, "Enhanced solar irradiance across the atmosphere-sea ice interface: A quantitative numerical study," Appl. 0pt. 44(13), 2613-2625 (2005).

[34] C. Moore, A. Barnard, P. Fietzek, M. R. Lewis, H. M. Sosik, S. White, and 0 . Zielinski, "Optical tools for ocean monitoring and research," ocean Sci. 5, 661-684 (2009). 